Gefässchirurgie 2015 $\cdot 20: 250-251$

DOI 10.1007/s00772-015-0038-1

Online publiziert: 3. Juni 2015

(c) Springer-Verlag Berlin Heidelberg 2015

\section{I.B. Kopp}

AWMF-Institut für Medizinisches Wissensmanagement, FB Medizin,

Philipps-Universität, Marburg, Deutschland

\title{
Akademische Gefäßchirurgie in Deutschland
}

\section{Betrachtung von klinischer Forschung, Leitlinien und Versorgungsforschung als Kontinuum mit dem Ziel der Verbesserung der Versorgungsqualität}

Der Chirurg, der die überwiegende Zeit seines Berufslebens im Operationssaal verbringt, wird durch die große Anzahl, den intensiven Gebrauch, aber auch den Missbrauch der Informations- und Aktionskanäle überwältigt - unter Bedingungen sich rasch entwickelnder Technologien und eines enorm gewachsenen ökonomischen Drucks [1]. Diese von Wilfried Lorenz vor über einem Vierteljahrhundert formulierte These scheint mehr als aktuell für die Situation der akademischen (Gefäß-)Chirurgie.

Die marktwirtschaftliche Orientierung des Gesundheitssystems macht Patienten zu Diagnoseträgern und fordert optimierte Betriebsabläufe [2]. In den letzten Jahren wurden allein über die Datenbank Medline pro Jahr über eine Million $\mathrm{Pu}$ blikationen erfasst, darunter etwa 10.000 pro Jahr zum Suchbegriff „vascular surgical procedures“. Die Publikationsflut ist unter dem Zeitdruck des klinischen Alltags kaum zu verfolgen und verhindert den Blick auf das Wesentliche - die eigentlich benötigte Information. Demgegenüber fehlen für viele, insbesondere chirurgische, Fragestellungen belastbare Erkenntnisse aus adäquat geplanten und durchgeführten klinischen Studien. Probleme der Verfügbarkeit und der Umsetzung vorhandenen Wissens werden als wesentliche Gründe der vielfach beobachteten regionalen Versorgungsunterschiede diskutiert. Man muss schon hinterfragen, warum z. B. in den USA die Revas- kularisationsraten bei $>65$ jährigen, gesetzlich versicherten Patienten mit PAVK je nach Krankenhauseinzugsgebiet bis um das Zehnfache variieren [3].

Leitlinien sollen hier eine Orientierung bieten und den Transfer relevanten Wissens in den Versorgungsalltag beschleunigen. Nach gängiger Definition sind Leitlinien „systematisch entwickelte Aussagen, die den gegenwärtigen Erkenntnisstand wiedergeben, um Ärzte und Patienten bei der Entscheidungsfindung für eine angemessene Versorgung in spezifischen klinischen Situationen zu unterstützen“ " 4 ]. Diese Definition impliziert zwei wesentliche Aspekte:

1. den Anspruch der Darlegung des aktuellen Stands der wissenschaftlichen Erkenntnisse für relevante Fragestellungen aufgrund der bestverfügbaren Evidenz aus klinischen Studien und der professionellen Erfahrung.

2. das Verständnis als Entscheidungshilfe für die Praxis, deren Anwendbarkeit in der individuellen Beratungssituation zwischen Arzt und Patient zu klären ist. Dabei spielen unter anderem die objektive Erfahrung, Kompetenz und Intuition des Arztes sowie Komorbidität, Erwartungen, Wertvorstellungen und die subjektive Erfahrung des Patienten mit seiner Erkrankung eine Rolle.

So verstanden und eingesetzt, dienen Leitlinien der Professionalisierung von Ärz- ten, der Stärkung von Patienten und der Verbesserung der Qualität der medizinischen Versorgung. Vor diesem Hintergrund engagieren sich die 171 Mitgliedsgesellschaften der AWMF (Arbeitsgemeinschaft der Wissenschaftlichen Medizinischen Fachgesellschaften e. V.) beträchtlich für die Entwicklung von Leitlinien und stellen diese kostenfrei über das Leitlinienregister der AWMF zur Verfügung (http://www.awmf.org/leitlinien. html). Von den etwa 700 Leitlinien im AWMF-Register adressieren etwa 30 unmittelbar gefäßchirurgisch relevante Themen.

\section{》) Leitlinien sollen eine Orientierung bieten und den Transfer relevanten Wissens in den Versorgungsalltag beschleunigen}

Leitlinienautoren haben die Aufgabe, das vorhandene Wissen unter methodischen und klinischen Gesichtspunkten darzulegen und zu werten. Sie nutzen dabei die Prinzipien und Vorgehensweisen der evidenzbasierten Medizin, aber auch der strukturierten Diskussion und Konsensfindung in einem für das Thema repräsentativen Gremium. Ziel ist es, die Expertise und Erfahrung verschiedener, von einer Leitlinie potenziell adressierter Disziplinen, Berufsgruppen und Patienten zu nutzen. Das Resultat ist keineswegs immer eine sichere Klärung der optimalen 
Vorgehensweise. Vielmehr werden auch Wissenslücken und Verbesserungspotenziale für künftige Studienplanungen erkannt und benannt, die für Forscher und Förderer unabhängiger Forschung relevant sind [5]. Leitlinienarbeit dient damit auch der Weiterentwicklung der Wissenschaftlichkeit eines Fachgebiets. Probleme von randomisierten klinischen Studien in der Gefäßchirurgie betreffen aus methodischer Sicht z. B. die geringen Fallzahlen, die heterogenen Patientenkollektive oder die Vergleichbarkeit der antithrombotischen Begleittherapie. Aus klinischer Sicht ist z. B. die erforderliche Qualifikation für Studienoperateure/Studieninterventionalisten und die Relevanz der Endpunkte (z. B. periprozedurale Ereignisse, MACCE, Langzeitüberleben, Lebensqualität) zu diskutieren. Wenn wir wissen wollen, welche Endpunkte tatsächlich patientenrelevant sind, müssen wir auch $\mathrm{Pa}$ tienten fragen.

Wer Leitlinien herausgibt, wird sich schließlich auch die Frage stellen, wie diese in der Praxis angenommen und angewendet werden und welche Auswirkungen sie tatsächlich auf die Versorgungsqualität haben. Analysen aus gut geführten Registern, Ergebnisse der externen vergleichenden Qualitätssicherung und epidemiologische Querschnittsstudien dienen dabei der Beobachtung der Versorgungssituation und der Frage nach dem Umsetzungsgrad bestimmter Leitlinienempfehlungen. Für andere Fragestellungen wird man andere Studiendesigns benötigen, z. B. Cluster-randomisierte Studien zur Untersuchung von Effekten gezielter Strategien zur Einführung von Leitlinien und qualitative Studien wie Fokusgruppenbefragungen zur Ermittlung von Barrieren gegen die Leitlinienumsetzung. Leitlinienimplementierung ist damit klassischer Gegenstand der Versorgungsforschung. Auch an dieser Stelle sind die Förderer unabhängiger Forschung gefragt.

Die Umsetzung von relevanten Forschungsergebnissen und Leitlinienempfehlungen muss aber von allen Akteuren, die das Gesundheitssystem gestalten - von Berufsverbänden und Patientenund Bürgervertretungen über die Selbstverwaltung bis zur Politik - mitgetragen werden. Dazu sind strategische Diskussionen und Partnerschaften erforderlich.

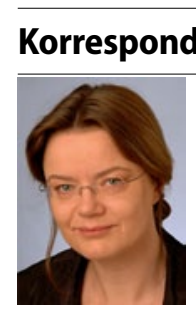

\section{Bildgebende Verfahren interaktiv dargestellt}

Für das Science Center Universum Bremen hat Fraunhofer MEVIS ein Exponat entwickelt, das die Methodenvielfalt moderner bildgebender Verfahren mit Hilfe des „Image Mans" einem breiten Publikum interaktiv vermittelt. Der „Image Man“ ist eine lebensgroße Figur, zusammengesetzt aus zehn Segmenten. Die Körperteile, die auf ihnen abgebildet sind, wurden mit unterschiedlichen Bildgebungsverfahren untersucht. Beispielsweise stammen die Aufnahmen von Wirbelsäule und Becken von einem CTScanner, um die Knochenstruktur sichtbar zu machen. Das Bild des Oberschenkels wurde per MR-Scanner erstellt, um Muskelfasern, Bindegewebe und Fett darzustellen. Die Bilder von Gehirn und Schilddrüse zeigen Stoffwechselaktivitäten, welche durch die Verfahren PET und SPECT ermittelt wurden. Im Zentrum der Installation steckt ein TouchScreen, mit dem sich der Besucher interaktiv durch die Resultate verschiedener Bildgebungsverfahren navigieren lassen kann. Darüber hinaus entwickeln Forscher von Fraunhofer MEVIS Lösungen für die klinische Routine, um Bilddaten, die aus verschiedenen Verfahren stammen oder zu unterschiedlichen Zeitpunkten aufgenommen wurden, zusammenzuführen. Die Programme ermöglichen beispielsweise eine Positionskorrelation der Bilddaten sowie eine Optimierung der Behandlungspläne, welche vor Beginn einer Strahlentherapie auf Basis einer CT-Aufnahme erstellt werden.

Quelle: Fraunhofer Mevis, www.mevis.fraunhofer.de 